Nonlinear Processes in Geophysics (2003) 10: 493-501

Nonlinear Processes

in Geophysics

(c) European Geosciences Union 2003

\title{
Conditional nonlinear optimal perturbation and its applications
}

\author{
M. Mu ${ }^{1}$, W. S. Duan ${ }^{1}$, and B. Wang ${ }^{2}$ \\ ${ }^{1}$ LASG, Institute of Atmospheric Physics, Chinese Academy of Sciences, Beijing 100029, China \\ ${ }^{2}$ Department of Meteorology, School of Ocean and Earth Science and Technology, University of Hawaii, Honolulu, USA
}

Received: 12 January 2001 - Revised: 23 July 2003 - Accepted: 13 October 2003

\begin{abstract}
Conditional nonlinear optimal perturbation (CNOP) is proposed to study the predictability of numerical weather and climate prediction. A simple coupled oceanatmosphere model for ENSO is adopted as an example to show its applicability. In the case of climatological mean state being the basic state, it is shown that CNOP tends to evolve into El Niño or La Niña event more probably than linear singular vector (LSV) on the condition that CNOP and LSV are of the same magnitude of norm. CNOP is also employed to study the prediction error of El Niño and La Niña events. Comparisons between CNOP and LSV demonstrate that CNOP is more applicable in studying the predictability of the models governing the nonlinear motions of oceans and atmospheres.
\end{abstract}

\section{Introduction}

Determination of the fastest growing initial perturbations in numerical weather and climate prediction and in the atmospheric research is of central importance. The linear approach for finding the fastest growing initial perturbation is widely adopted in both the theoretical studies and the numerical weather prediction. Usually, it is assumed that the initial perturbation is sufficiently small such that its evolution can be governed approximately by the tangent linear model (TLM) of the nonlinear model. For a discrete TLM, the forward propagator can be expressed as a matrix, and computing the linear fastest growing perturbation is reduced to calculate the linear singular vector (LSV), which corresponds to the maximum singular value of the matrix. LSV and linear singular value (LSVA) were introduced into meteorology by Lorenz (1965) to investigate the predictability of the atmospheric motion. Buizza and Palmer (1995) utilized LSVs to study the patterns of the atmospheric general circulations. Recently, this method has been used to find out the initial condition for optimal growth in a coupled ocean-

Correspondence to: M. $\mathrm{Mu}$ (mumu@lasg.iap.ac.cn) atmosphere model of El Niño-Southern Oscillation (ENSO), in an attempt to explore error growth and predictability of the coupled model (Xue and Cane, 1997a, b; Thompson, 1998; Samelson and Tziperman, 2001). In addition, LSVs are employed in the ensemble numerical weather prediction. At the European Center for Medium-Range Weather Forecasts (ECMWF), LSVs are utilized to construct the initial perturbations for the ensemble forecast, in order to estimate the probability distribution of the forecast states.

The motions of the atmosphere and ocean are governed by complicated nonlinear systems. The theory of LSV and LSVA is established on the basis that the evolution of the initial perturbation can be described approximately by the linearized version of the nonlinear model. This raises a few questions concerning the validity of TLM. One is how small the initial perturbation should be to guarantee the validity of TLM; another is how to determine the time interval during which the TLM is valid. There has been a few papers addressing these concerns, but no satisfying answer has been given (Lacarra and Talagrand, 1988; Tanguay and Bartello, 1995; Mu et al., 2000). Therefore, for the nonlinear systems in the numerical weather and climate prediction, it is desirable and often necessary to deal with the nonlinear models themselves rather than their linear approximations. Leading Lyapunov vector and exponent have also been used to study predictability problems (Lorenz, 1996). Realizing that when the initial uncertainty is not very small, the leading Lyapunov exponent may not be a good measurement of the predictability, Aurell et al. (1997) introduced the concept of finite size Lyapunov exponent, which has been applied by Boffetta et al. (1998) to study the predictability of the atmosphere. Toth and Kalnay (1997) also pointed out that breeding method, which has been used to generate initial perturbations in ensemble prediction, provides an extension of the concept of Lyapunov vector into the nonlinear field with finite amplitude perturbations. Oortwijn and Barkmeijer (1995) and Barkmeijer (1996) also realized the limitation of TLM and considered the nonlinear effects by an iterative procedure.

A novel concept of nonlinear singular value (NSVA) and 
nonlinear singular vector (NSV) has been formulated, which is a natural generalization to the classical LSVA and LSV (Mu, 2000). The approach of Oortwijn and Barkmeijer (1995) and Barkmeijer (1996) consists of modifying the linear approach by a numerical iterative procedure. Hence the comparison between their results and ones obtained by NSV can only be made numerically. Since the focus of this paper is not NSV, such comparison is left to the future. Let $\boldsymbol{U}=\boldsymbol{U}(x, t)$ be the basic state and $\boldsymbol{U}_{0}=\boldsymbol{U}(x, 0)$ its initial state. If $\boldsymbol{u}_{0}(x)$ is an initial perturbation superposed on $\boldsymbol{U}_{0}, \boldsymbol{U}_{0}+\boldsymbol{u}_{0}$ will evolve into $\boldsymbol{U}(x, T)+\boldsymbol{u}(x, T)$ at time $T$, where $\boldsymbol{u}(x, T)$ describes the nonlinear evolution of the initial perturbation $\boldsymbol{u}_{0}(x)$. The initial perturbation $\boldsymbol{u}_{0}^{*}$ is called the fastest nonlinear growing perturbation, or the first NSV with respect to the basic state $\boldsymbol{U}(x, t)$ in terms of the norm $\|\cdot\|$, if and only if

$$
I\left(\boldsymbol{u}_{0}^{*}\right)=\max _{\boldsymbol{u}_{0}} I\left(\boldsymbol{u}_{0}\right)
$$

where

$I\left(\boldsymbol{u}_{0}\right)=\frac{\|\boldsymbol{u}(T)\|^{2}}{\left\|\boldsymbol{u}_{0}\right\|^{2}}$.

The square root of Eq. (1), called the first NSVA, is the largest growth rate of perturbation $\boldsymbol{u}(t)$ at time $T$. In addition to the first NSVA and NSV, we can also define the $n$th $(n=1,2, \cdots)$ NSVA and NSV (Mu, 2000). Durbiano (2001) successfully computed the first six NSVs of a shallow water model.

The two-dimensional quasi-geostrophic model has been also used to study the NSVA and NSV (Mu and Wang, 2001). The numerical results demonstrate that for some types of basic states, there exists $u_{0}^{*}$ such that in the phase space functional $I\left(\boldsymbol{u}_{0}\right)$ attains local maximum at $u_{0}^{*}$, which is called local fastest growing perturbations. But there is no such phenomenon in the case of LSVs and LSVAs due to the absence of the nonlinearity of the corresponding TLM. The local fastest growing perturbations usually possess larger norms comparing to the first NSV, which corresponds to the global maximum value of functional $I\left(\boldsymbol{u}_{0}\right)$. Although the growth rates of the local fastest growing perturbations are smaller than the first NSVA, their nonlinear evolutions at the end of the time interval are considerably greater than that of the first NSV in terms of the chosen norm. In this case, the local fastest growing perturbations could play a more important role than the global fastest growing perturbation in the study of the predictability.

It is clear from the results of Mu and Wang (2001) that for predictability study, we should first find out all local fastest growing perturbations, then investigate their effects on the predictability. This is inconvenient in practical application. Besides, sometimes the local fastest growing perturbation with a large norm could be physically unreasonable. For instance, in an anomaly model of ENSO system, sea surface temperature (SST) anomaly usually have an upper bound. For example, over the period 1900-1999, SST anomaly averaged over Niño-3 region is always less than $6^{\circ} \mathrm{C}$. Hence, the initial perturbation (initial SST anomaly) superposed on the climatological mean state should be less than this bound. If one neglects this constraint, the local fastest growing perturbations with a large norm arising from a numerical approach could exceed this bound and become unreasonable initial SST anomaly.

Finally, when we compute the NSVs and NSVAs, the inequality $\|\boldsymbol{u}(T)\| \leq c\left\|\boldsymbol{u}_{0}\right\|$ must be satisfied, where $\boldsymbol{u}_{0}$ is an arbitrary initial perturbation, $\boldsymbol{u}(T)$ is its nonlinear evolution and $c$ is a constant independent of $\boldsymbol{u}_{0}(\mathrm{Mu}, 2000)$. However it is difficult to check this requirement for complicated governing equations of atmosphere and ocean.

All these weaknesses suggest that we should investigate the nonlinear optimal perturbation with constrained conditions. This paper is devoted to address this problem.

The paper is organized as follows. In Sect. 2, we present the concept of conditional nonlinear optimal perturbation. In Sect. 3 we give a brief description of general-purpose sequential quadratic programme (SQP) algorithm, which is used in this paper to compute CNOP. In Sect. 4, a simple coupled ocean-atmosphere model for ENSO is adopted as an example to investigate the applications of CNOP. The conclusion and discussion are presented in Sect. 5.

\section{Conditional nonlinear optimal perturbation}

Assume that the model governing the motions of the atmosphere or ocean is as follows:

$\left\{\begin{array}{l}\frac{\partial \boldsymbol{w}}{\partial t}+F(\boldsymbol{w})=0, \quad \text { in } \Omega \times[0, T] \\ \left.\boldsymbol{w}\right|_{t=0}=\boldsymbol{w}_{0},\end{array}\right.$

where $\boldsymbol{w}(\boldsymbol{x}, t)=\left(w_{1}(\boldsymbol{x}, t), w_{2}(\boldsymbol{x}, t), \ldots, w_{n}(\boldsymbol{x}, t)\right), F$ a nonlinear operator, and $\boldsymbol{w}_{0}$ the initial state, $(\boldsymbol{x}, t) \in \Omega \times[0, T]$, $\Omega$ a domain in $R^{n}$, and $T<+\infty, \boldsymbol{x}=\left(x_{1}, x_{2}, \ldots, x_{n}\right), t$ the time. To facilitate the following discussion, suppose initial value problem (2.1) is well-posed and $M$ is the propagator from 0 to time $T$. Hence, for fixed $T>0$, the solution $\boldsymbol{w}(\boldsymbol{x}, T)=M\left(\boldsymbol{w}_{0}\right)(T)$ is well-defined. Let $\boldsymbol{U}(\boldsymbol{x}, t)$ and $\boldsymbol{U}(\boldsymbol{x}, t)+\boldsymbol{u}(\boldsymbol{x}, t)$ be the solutions of problem (2.1) with initial value $\boldsymbol{U}_{0}$ and $\boldsymbol{U}_{0}+\boldsymbol{u}_{0}$ respectively, where $\boldsymbol{u}_{0}$ is the initial perturbation. We have

$\boldsymbol{U}(T)=M\left(\boldsymbol{U}_{0}\right)(T), \quad \boldsymbol{U}(T)+\boldsymbol{u}(T)=M\left(\boldsymbol{U}_{0}+\boldsymbol{u}_{0}\right)(T)$

So $\boldsymbol{u}(T)$ describes the evolution of the initial perturbation $\boldsymbol{u}_{0}$.

For a chosen norm $\|\cdot\|$ measuring $\boldsymbol{u}$, the perturbation $\boldsymbol{u}_{0 \delta}$ is called the conditional nonlinear optimal perturbation with constraint condition $\left\|\boldsymbol{u}_{0}\right\| \leq \delta$, if and only if

$J\left(\boldsymbol{u}_{0 \delta}\right)=\max _{\left\|\boldsymbol{u}_{0}\right\| \leq \delta} J\left(\boldsymbol{u}_{0}\right)$

where

$J\left(\boldsymbol{u}_{0}\right)=\left\|M\left(\boldsymbol{U}_{0}+\boldsymbol{u}_{0}\right)(T)-M\left(\boldsymbol{U}_{0}\right)(T)\right\|$.

In the above, the constraint condition is simply expressed as belonging to a ball with chosen norm. Obviously, we can also investigate the situations that the perturbations belong 
to other kind of functional set. Furthermore, the constraint condition could be some physical laws, which the perturbation should satisfy.

In this paper, we adopt a sequential quadratic programming (SQP) solver to compute CNOP, which is described in Sect. 3 .

\section{Description of SQP method}

After discretization and proper transformation of the objective function, the nonlinearly constrained optimization problem considered in this paper can be written in the form

$\min _{\boldsymbol{x} \in R^{n}} F(\boldsymbol{x})$,

subject to

$\boldsymbol{h}(\boldsymbol{x}) \leq 0$,

where $\boldsymbol{h}=\left(h_{1}, h_{2}, \cdots, h_{n}\right)^{\top}$ is a vector of nonlinear functions. It is assumed that at any point $\boldsymbol{x}$ the gradient $\boldsymbol{\nabla} F(\boldsymbol{x})$ of the objective function and the Jacobian $\mathbf{J}(\mathbf{x})=\frac{\partial\left(h_{1}, h_{2}, \cdots, h_{n}\right)}{\partial\left(x_{1}, x_{2}, \cdots, x_{n}\right)}$ of constraint function can be computed.

SQP is a class of optimization solvers that can be used to solve the nonlinear minimization problems with equality and inequality constraint condition. The SQP algorithm described in Powell (1982) is as follows.

Step 0. Set iteration $i=0$, a solution guess $\boldsymbol{x}^{0}$, a Hessian Lagrangian estimate $\mathbf{H}^{\mathbf{0}}=\mathbf{I}$, which is the identity matrix, and an initial given value of Lagrange multiplier, $\lambda^{0}$.

Step 1. Evaluate the objective functions and their gradients.

$F\left(\boldsymbol{x}^{i}\right), \quad \boldsymbol{h}\left(\boldsymbol{x}^{i}\right)$

$\nabla F\left(\boldsymbol{x}^{i}\right), \nabla \boldsymbol{h}\left(\boldsymbol{x}^{i}\right)$

Step 2. Compute $\boldsymbol{d}^{i}$ by the following quadratic programme (QP) sub-problem,

$\min _{\boldsymbol{d}}\left(\left[\nabla F\left(\boldsymbol{x}^{i}\right)\right]^{\top} \boldsymbol{d}^{i}+\frac{1}{2}\left(\boldsymbol{d}^{i \top} \mathbf{H}^{i} \boldsymbol{d}^{i}\right)\right.$,

subject to

$\boldsymbol{h}\left(\boldsymbol{x}^{i}\right)+\left[\nabla \boldsymbol{h}\left(\boldsymbol{x}^{i}\right)\right]^{\top} \boldsymbol{d}^{i} \leq 0$,

where $\boldsymbol{d}^{i}$ is a direction of descent for the objective function. Then using $\boldsymbol{d}^{i}$, we determine the Lagrange multiplier $\lambda^{i+1}$ corresponding to the QP sub-problem (Barclay et al., 1997). Step 3. Check convergence. If $\boldsymbol{x}^{i}, \lambda^{i+1}$ satisfy $\left\|\boldsymbol{\nabla} \boldsymbol{L}\left(\boldsymbol{x}^{i}, \lambda^{i+1}\right)\right\| \leq \epsilon$, where $\boldsymbol{\nabla} \boldsymbol{L}=\boldsymbol{\nabla} F+\nabla \boldsymbol{h} \lambda$, and $\epsilon$ is a given positive number to guarantee the convergence, then $\boldsymbol{x}^{i}$ is the point at which the objective $F(\boldsymbol{x})$ is minimal. Otherwise, let $\boldsymbol{x}^{i+1}=\boldsymbol{x}^{i}+\alpha \boldsymbol{d}^{i}, \alpha \leq 1$, and then go to Step 4.

Step 4. Update Hessian Lagrangian. Let $\boldsymbol{s}^{i}=\boldsymbol{x}^{i+1}-\boldsymbol{x}^{i}$, and $\boldsymbol{y}^{i}=\nabla \boldsymbol{L}\left(\boldsymbol{x}^{i+1}, \lambda^{i+1}\right)-\nabla \boldsymbol{L}\left(\boldsymbol{x}^{i}, \lambda^{i}\right)$. The new Hessian Lagrangian, $\mathbf{H}^{i+1}$, can be obtained by calculating

$\mathbf{H}^{i+1}=\mathbf{H}^{i}-\frac{\mathbf{H}^{i} \boldsymbol{s}^{i} \boldsymbol{s}^{i \top} \mathbf{H}^{i \top}}{\boldsymbol{s}^{i \top} \mathbf{H}^{i} \boldsymbol{s}^{i}}+\frac{\boldsymbol{y}^{i} \boldsymbol{y}^{i \top}}{\boldsymbol{y}^{i \top} \boldsymbol{s}^{i}}$.

Then go to Step 2.

\section{Applications of conditional nonlinear optimal pertur- bations to a simple coupled ocean-atmosphere model for ENSO}

In this section, the CNOP is applied to study the predictability of ENSO within the framework of the simple coupled ocean-atmosphere model of Wang et al. (1999). The ocean component of this theoretical model is derived from the model of Zebiak and Cane (1987). The essence of the ocean component is the nonlinear coupling between the mixed layer thermodynamics and the upper ocean dynamics. The wind forcing required by ocean component is described by diagnostic equations based on a simplified Lindzen-Nigam model (Lindzen and Nigam, 1987). By focusing on the equatorially trapped east-west seesaw structure of the ENSO, this simple coupled model is formulated using Lorenz (1963) truncation in terms of two first-order nonlinear ordinary differential equations. The two dimensionless equations describe, respectively, the time evolution of the anomalous sea surface temperature $T_{E}$ and the anomalous thermocline depth $h_{E}$ both in the equatorial eastern Pacific:

$\left\{\begin{array}{l}\frac{d T_{E}}{d t}=a_{1} T_{E}+a_{2} h_{E}+\sqrt{\frac{2}{3}} T_{E}\left(T_{E}-\mu h_{E}\right)-2 T_{E}^{3}, \\ \frac{d h_{E}}{d t}=b\left(2 h_{E}-T_{E}\right)-h_{E}^{3},\end{array}\right.$

where

$a_{1}=\left(\Delta \bar{T}_{z}+\Delta \bar{T}_{x}-\alpha_{s}\right)$,

$a_{2}=-\mu \Delta \bar{T}_{x}$,

$b=\frac{2 \alpha}{p\left(1-3 \alpha^{2}\right)}$.

The coefficients $a_{1}$ and $a_{2}$ involve basic state parameters $\Delta \bar{T}_{x}$ and $\Delta \bar{T}_{z}$, which characterize, respectively, the mean temperature difference between the east and west and between the surface and subsurface water. Note that, these basic state parameters vary with time, reflecting the annual cycle of the basic state. $\alpha_{s}$ is a Newtonian cooling coefficient for SST anomalies. The coefficient $\mu$ measures the effect of the thermocline displacement on SST. $\alpha$ is the air-sea coupling coefficient. $p$ is a function of meridional length scales. For more detailed description of the simple coupled model, the readers are referred to Wang and Fang (1996) and Wang et al. (1999).

The steady solution $(0,0)$ represents the climatological mean equilibrium state or an ENSO "transitional" state (including annul cycle) in which both SST and the depth of thermocline are normal.

The model is integrated by fourth-order Runge-Kutta scheme with $d t=0.01$, which represents one day. The Fortran code of SQP adopted is a modified version of Powell (1982) and can be gotten from Y. Yuan (e-mail: yyx@1sec.cc.ac.cn).

4.1 Conditional nonlinear optimal perturbation of $O-$ the climatological mean equilibrium state

In this paper, the norm $\left\|\boldsymbol{u}_{0}\right\|=\sqrt{T_{E 0}{ }^{2}+h_{E 0}^{2}}$ is employed to measure the perturbation $\boldsymbol{u}(t)$, where $T_{E 0}$ and $h_{E 0}$ 


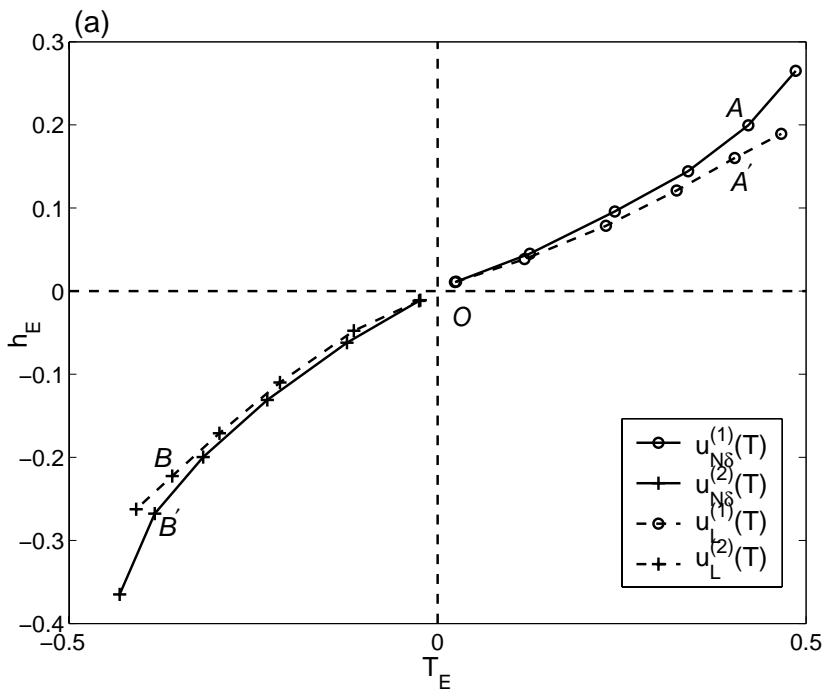

(b)

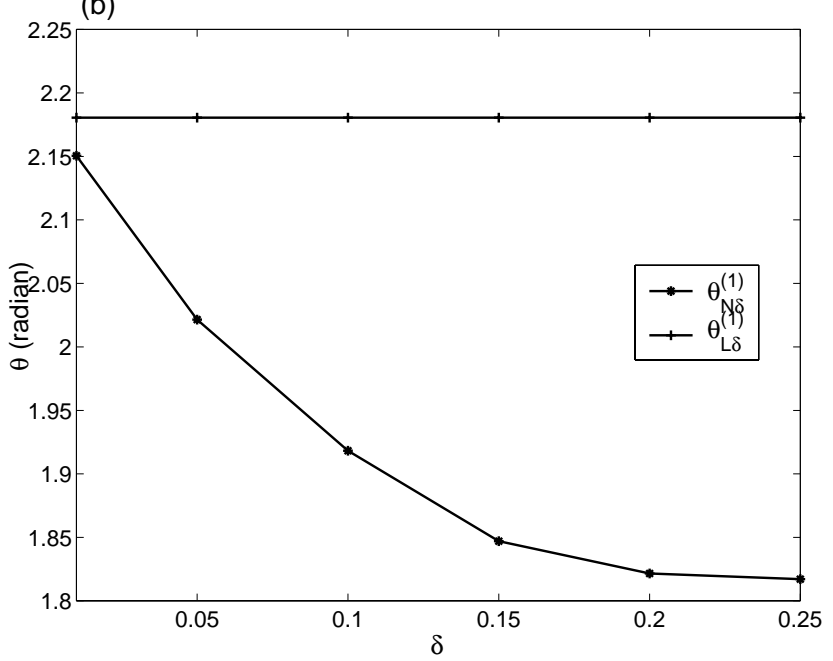

Fig. 1. (a) $\boldsymbol{u}_{N \delta}^{(i)}(T)$ and $\boldsymbol{u}_{L}^{(i)}(T)$, the nonlinear evolutions of $\boldsymbol{u}_{0 \delta}^{(i)}$ and $\boldsymbol{u}_{0 L}^{(i)}$ in the phase space respectively; (b) $\theta_{L \delta}^{(1)}$ and $\theta_{N \delta}^{(1)}$, the position angles of $\boldsymbol{u}_{0 L}^{(1)}$ and $\boldsymbol{u}_{0 \delta}^{(1)}$ as functions of $\delta$, respectively.

represent the initial SST and thermocline depth anomaly, respectively. For $T=10,12$ months with initial time being January, we obtained CNOPs of basic state $O$ with constraint condition $\left\|\boldsymbol{u}_{0}\right\| \leq \delta, \delta \in[0.01,0.25]$ respectively. The results for $T=10,12$ months are quite similar. For simplicity, we only show that of the case of $T=12$ months. In this case, there exist two different CNOPs, $\boldsymbol{u}_{0 \delta}^{(1)}$ and $\boldsymbol{u}_{0 \delta}^{(2)}$, which are all on the boundary of the corresponding disc $\left\|\boldsymbol{u}_{0}\right\| \leq \delta, \delta \in[0.01,0.25]$. Let $\theta_{N \delta}^{(i)}, i=1,2$, be the position angles of the two CNOPs, which is the one between the CNOP and the positive $T_{E}$-axis in the plane $T_{E}-h_{E}$ and represents the direction of the vector $\boldsymbol{u}_{0 \delta}^{(i)}$. Then the two CNOPs can be expressed as $\boldsymbol{u}_{0 \delta}^{(1)}=\left(\delta \cos \theta_{N \delta}^{(1)}, \delta \sin \theta_{N \delta}^{(1)}\right)$ and $\boldsymbol{u}_{0 \delta}^{(2)}=\left(\delta \cos \theta_{N \delta}^{(2)}, \delta \sin \theta_{N \delta}^{(2)}\right)$ respectively, where $\delta$ is the magnitude of initial perturbation. For $\delta=0.20$, the two CNOPs are $(-0.0498,0.1937)$ and $(0.0295,-0.1978)$ and located in (a)

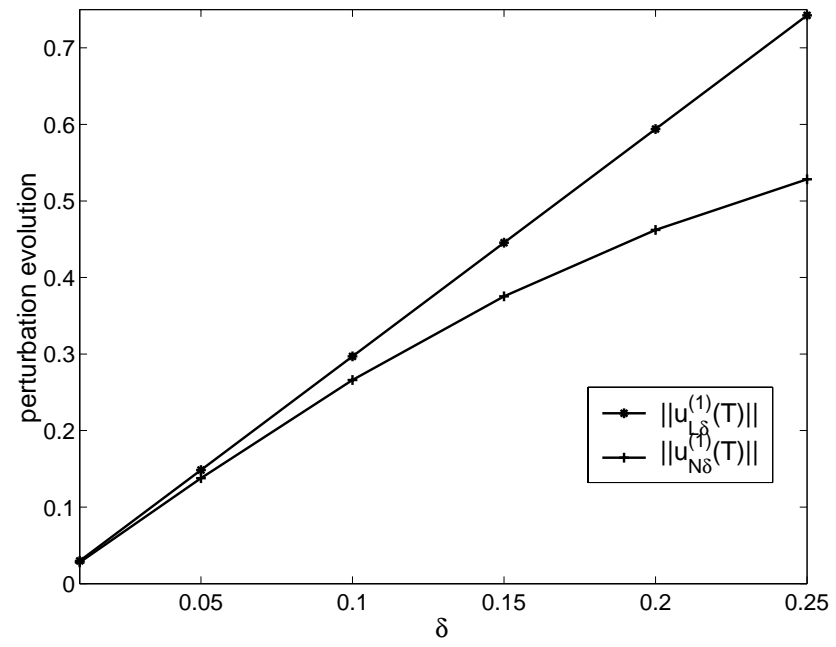

(b)

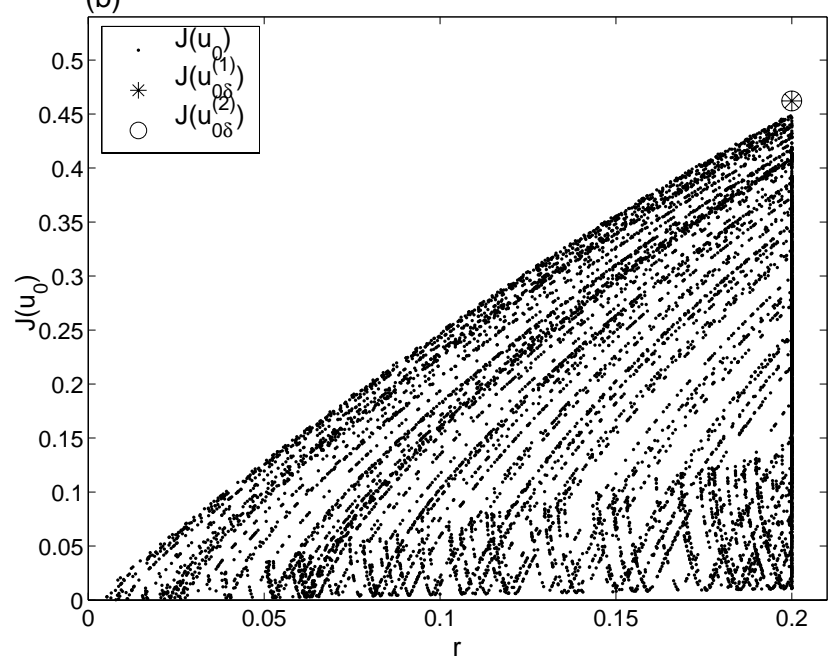

Fig. 2. (a) $\left\|\boldsymbol{u}_{N \delta}^{(1)}(T)\right\|$ and $\left\|\boldsymbol{u}_{L \delta}^{(1)}(T)\right\|$, the nonlinear and linear evolutions of $\boldsymbol{u}_{0 \delta}^{(1)}$ with $T=12$ months respectively; (b) $J\left(\boldsymbol{u}_{0}\right)$ for random initial perturbations in disk $\left\|\boldsymbol{u}_{0}\right\| \leq 0.20, J\left(\boldsymbol{u}_{0 \delta}^{(1)}\right)$ and $J\left(\boldsymbol{u}_{0 \delta}^{(2)}\right)$.

II and IV-Quadrant respectively. To further investigate the difference between the two CNOPs, we integrate the model with initial values $\boldsymbol{u}_{0 \delta}^{(1)}$ and $\boldsymbol{u}_{0 \delta}^{(2)}$ for each $\delta \in[0.01,0.25]$ and obtain their nonlinear developments, $\boldsymbol{u}_{N \delta}^{(1)}(T)$ and $\boldsymbol{u}_{N \delta}^{(2)}(T)$ for $T=12$ months. The results are plotted in Fig. 1a. It is readily shown that with $\delta$ increasing from 0.01 to 0.25 , $\boldsymbol{u}_{N \delta}^{(1)}(T)\left(\boldsymbol{u}_{N \delta}^{(2)}(T)\right)$ departs from the neighborhood of $O$ and the two different CNOPs evolve into the patterns located in IQuadrant and III-Quadrant in the plane $T_{E}-h_{E}$ respectively.

To compare CNOP with LSV, we further investigate the nonlinear evolution and the directions of LSV. $\boldsymbol{u}_{0 L}=$ $(-0.0573,0.0819)$ is a LSV of the basic state $O$ and located in II-Quadrant. To facilitate the following discussion, we define two scaled LSVs,

$\boldsymbol{u}_{0 L}^{(1)}=\frac{\left\|\boldsymbol{u}_{0 \delta}^{(1)}\right\|}{\left\|\boldsymbol{u}_{0 L}\right\|} \boldsymbol{u}_{0 L}, \quad \boldsymbol{u}_{0 L}^{(2)}=-\frac{\left\|\boldsymbol{u}_{0 \delta}^{(2)}\right\|}{\left\|\boldsymbol{u}_{0 L}\right\|} \boldsymbol{u}_{0 L}$, 
thus

$\left\|\boldsymbol{u}_{0 L}^{(1)}\right\|=\left\|\boldsymbol{u}_{0 \delta}^{(1)}\right\|=\delta, \quad\left\|\boldsymbol{u}_{0 L}^{(2)}\right\|=\left\|\boldsymbol{u}_{0 \delta}^{(2)}\right\|=\delta$.

Integrating the nonlinear model with $\boldsymbol{u}_{0 L}^{(1)}$ and $\boldsymbol{u}_{0 L}^{(2)}$ being initial values, we obtain the evolutions, $\boldsymbol{u}_{L}^{(i)}(T)$, for each $\delta \in[0.01,0.25]$ respectively. The results are also plotted in Fig.1a, where $A$ and $A^{\prime}$ correspond to the nonlinear evolutions of $\boldsymbol{u}_{0 \delta}^{(1)}$ and $\boldsymbol{u}_{0 L}^{(1)}$ with $\delta=0.2, B$ and $B^{\prime}$ to the ones of $\boldsymbol{u}_{0 \delta}^{(2)}$ and $\boldsymbol{u}_{0 L}^{(2)}$ with $\delta=0.2$ respectively. It is easily shown that for the same value of $\delta$, when it is large, for example, $\delta=0.2, \boldsymbol{u}_{N \delta}^{(1)}(T)\left(\boldsymbol{u}_{N \delta}^{(2)}(T)\right)$ is quite different from $\boldsymbol{u}_{L}^{(1)}(T)$ $\left(\boldsymbol{u}_{L}^{(2)}(T)\right)$. And with $\delta$ increasing from 0.01 to 0.25 , the differences become more and more considerable. Besides, $\boldsymbol{u}_{0 L}^{(i)}$ can also be expressed as $\left(\delta \cos \theta_{L \delta}^{(i)}, \delta \sin \theta_{L \delta}^{(i)}\right)$, where $\theta_{L \delta}^{(i)}$, entitled position angle in this paper, is the one between LSV and the positive $T_{E}$-axis in the plane $T_{E}-h_{E}$ and represents the direction of the LSV $u_{0 L}^{(i)}$. The position angles of CNOP $u_{0 \delta}^{(1)}$ and LSV $u_{0 L}^{(1)}$ are shown in Fig. 1b. It is demonstrated that the direction of LSV does not change with $\delta$, but those of CNOPs do. This indicates that LSV represents the optimal growing direction due to the linearity of tangent linear model (Mu and Wang, 2001), but CNOP stands for initial pattern, whose nonlinear evolution is maximal at time $T$. Figure $2 \mathrm{a}$ are the nonlinear and linear evolutions of $\boldsymbol{u}_{0 \delta}^{(1)}$. It follows that there are remarkable differences between them for large perturbations. However, when the initial perturbations are sufficiently small, the difference becomes trivial. Meanwhile, the CNOP tends gradually to the LSV (Fig. 1b). This demonstrates that for large initial perturbations, the tangent linear model is not a good approximation to the nonlinear model.

To verify the correctness of our optimization algorithm, for $\boldsymbol{u}_{0 \delta}^{(1)}$ and $\boldsymbol{u}_{0 \delta}^{(2)}$ with $\delta=0.20$, a large random samples of initial perturbations in the disk $\left\|\boldsymbol{u}_{0}\right\| \leq 0.20$, are chosen to find out the maximum value of functional $J\left(\boldsymbol{u}_{0}\right)$ (Fig. 2b). It is clear from Fig. $2 \mathrm{~b}$ that the value of $J\left(\boldsymbol{u}_{0}\right)$ with other random perturbations in the disk $\left\|\boldsymbol{u}_{0}\right\| \leq 0.20$ is always less than $J\left(\boldsymbol{u}_{0 \delta}^{(i)}\right), i=1,2$. This verifies that the CNOPs for $\delta=0.20$ are indeed the nonlinear optimal perturbations with constraint condition $\left\|\boldsymbol{u}_{0}\right\| \leq 0.20$. For the other values of $\delta$, $\delta \in[0.01,0.25]$, the results are similar to that of $\delta=0.20$. For simplicity, only the case of $\delta=0.20$ is shown. These indicate that SQP algorithm adopted in this paper can solve efficiently the optimization problem Eq. (4).

The CNOPs of basic state $O$ are the initial perturbations superposed on the climatological mean state, which represent the initial anomalies $\left(T_{E}, h_{E}\right)$. In this model, if $T_{E} \geq 0.25$ $\left(T_{E} \leq-0.25\right)$ persists for more than three months, it is regarded as an El Niño (La Niña) event. Let $\boldsymbol{u}_{N \delta}^{(i)}(t)$ be the evolution of $\boldsymbol{u}_{0 \delta}^{(i)}$, we plot in Fig. 3 the $T_{E}$ component of it. According to this standard, for $T=12$ months, when $\delta \geq 0.10(\delta \geq 0.12)$, the CNOP $\boldsymbol{u}_{0 \delta}^{(1)}\left(\boldsymbol{u}_{0 \delta}^{(2)}\right)$ of the basic state $O$ will evolve into El Niño (La Niña) event (Fig. 3). Then what about the evolution of LSV?
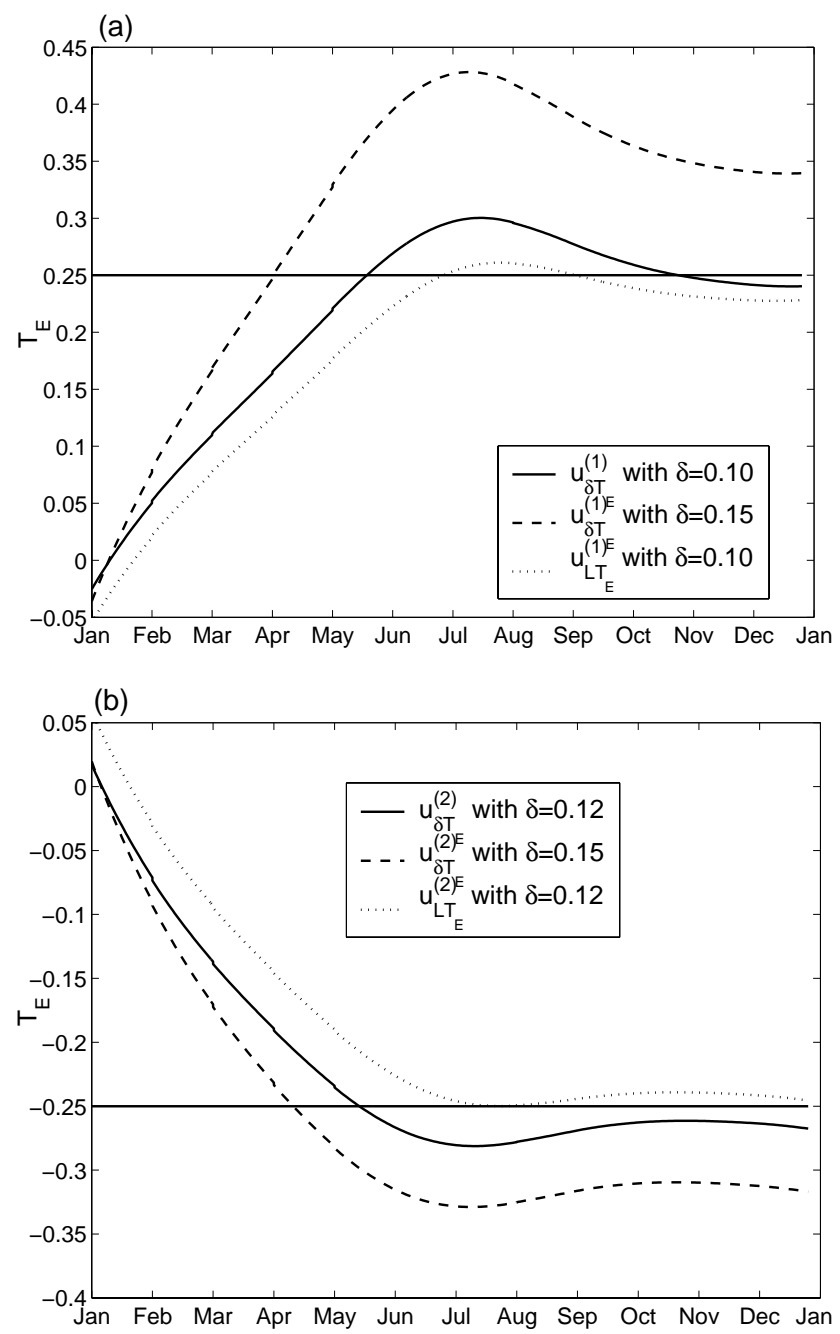

Fig. 3. $u_{\delta T_{E}}^{(i)}(t)$ and $u_{L T_{E}}^{(i)}(t)$, the $T_{E}$ components of $\boldsymbol{u}_{N \delta}^{(i)}(t)$ and $\boldsymbol{u}_{L}^{(i)}(t), i=1,2$, respectively.

Taking the scaled LSVs, $\boldsymbol{u}_{0 L}^{(1)}$ with $\delta=0.10$ and $\boldsymbol{u}_{0 L}^{(2)}$ with $\delta=0.12$, as initial values, integrating the nonlinear model respectively, we obtain the evolutions of $\boldsymbol{u}_{L}^{(i)}(t)$, of which the $T_{E}$ component are shown in Fig. 3 too. The results demonstrate that $\boldsymbol{u}_{0 L}^{(1)}$ with $\delta=0.10\left(\boldsymbol{u}_{0 L}^{(2)}\right.$ with $\left.\delta=0.12\right)$ does not evolve into El Niño (La Niña) event. In the prediction and research of ENSO, it is important to find out the initial patterns which will evolve into El Niño or La Niña event most probably (Thompson, 1998). Our results suggest that CNOP is more applicable than LSV for this purpose.

In the following subsections, we investigate the CNOPs of an El Niño and a La Niña events and their applications to the estimation of prediction error of El Niño and La Niña events.

\subsection{Estimation of prediction errors of El Niño and La Niña events, $\boldsymbol{U}^{(1)}(t)$ and $\boldsymbol{U}^{(2)}(t)$}

In this subsection, the basic states $\boldsymbol{U}^{(1)}(t)$ and $\boldsymbol{U}^{(2)}(t)$ are the El Niño and La Niña events, which are obtained 
(a)

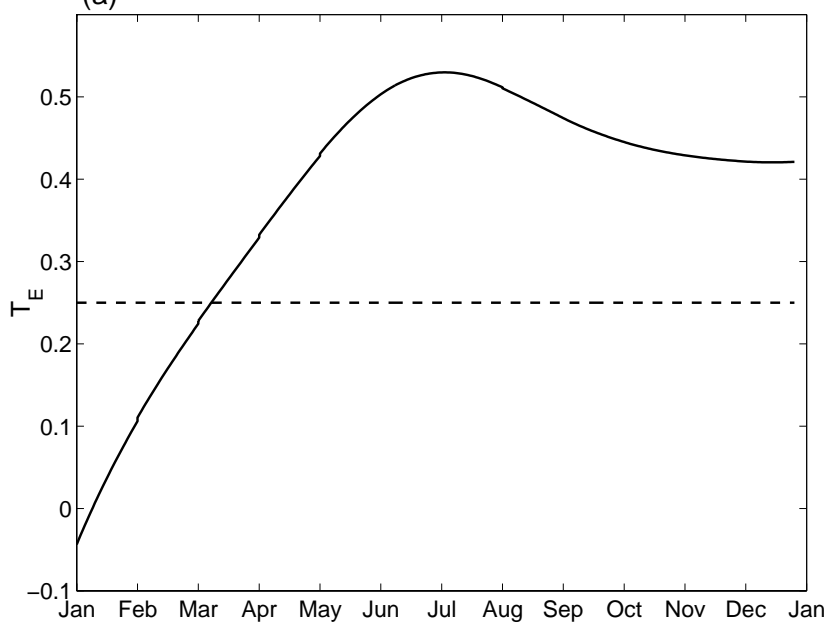

(b)

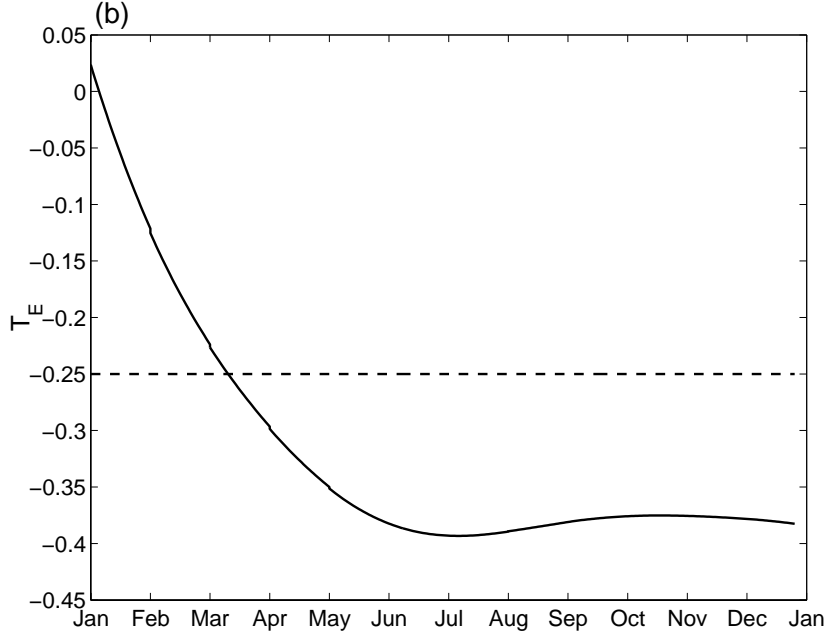

Fig. 4. (a) Basic state $\boldsymbol{U}^{(1)}(t)$. (b) Basic state $\boldsymbol{U}^{(2)}(t)$.

by integrating nonlinear model with initial values $\boldsymbol{u}_{0 \delta}^{(1)}=$ $(-0.0498,0.1937)$ and $\boldsymbol{u}_{0 \delta}^{(2)}=(0.0295,-0.1978)$ respectively, which are the two CNOPs of the climatological mean state with $\delta=0.20$ and $T=12$ months obtained in last subsection (Fig. 4).

Assume that the initial values of the basic states, $\boldsymbol{u}_{0 \delta}^{(1)}$ and $\boldsymbol{u}_{0 \delta}^{(2)}$, are taken as initial observations, then the CNOPs of $\boldsymbol{U}^{(1)}(t)$ and $\boldsymbol{U}^{(2)}(t)$ are closely related to the problem of the maximum prediction error. We will explain it in detail. Mu et al. (2002) classified the predictability problems in numerical weather and climate prediction into three problems, which are, respectively, the maximum predictable time, the maximum prediction error, and the maximum admissible errors of the initial values and the parameters in the model. Suppose that $M$ is the propagator from time 0 to $T, \boldsymbol{U}_{0}$ the initial observation, the prediction error is

$E=\left\|M\left(\boldsymbol{U}_{0}\right)(T)-\boldsymbol{U}_{T}^{t}\right\|$,

where $\boldsymbol{U}_{T}^{t}$ is the true value of the state at time $T$. Since the true value $\boldsymbol{U}_{T}^{t}$ can not be obtained exactly, it is impossible
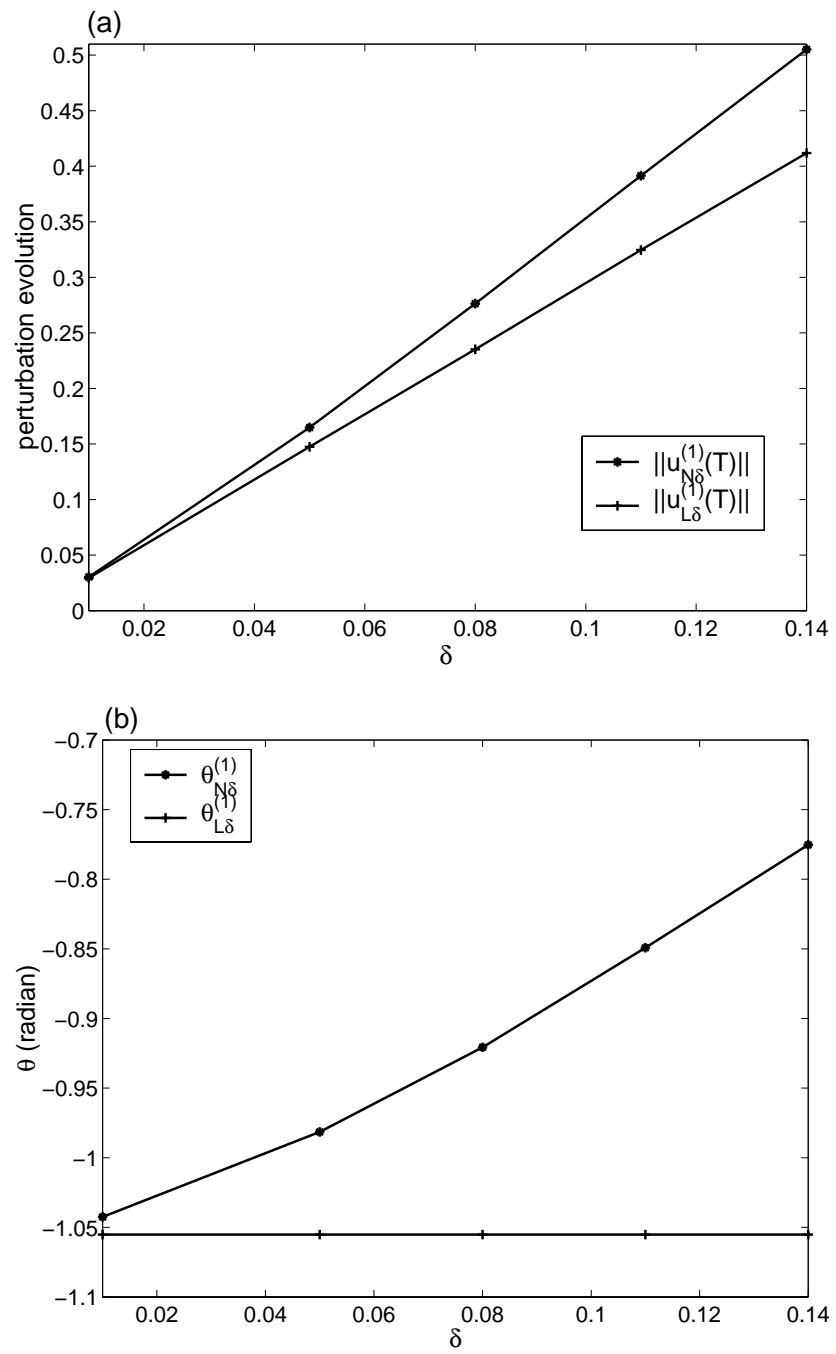

Fig. 5. (a) $\left\|\boldsymbol{u}_{N \delta}^{(1)}(T)\right\|$ and $\left\|\boldsymbol{u}_{L \delta}^{(1)}(T)\right\|$, the nonlinear and linear evolution of CNOP of $\boldsymbol{U}^{(1)}(t)$ for $T=10$ months and $\delta \in[0.01,0.14]$; (b) $\theta_{N \delta}^{(1)}$ and $\theta_{L \delta}^{(1)}$, the position angles of LSV and CNOP of $\boldsymbol{U}^{(1)}$ as functions of $\delta$, respectively.

to get the exact value of $E$. But Mu et al. (2002) pointed out that if some information about the errors of the initial observation is known, e.g., if we know that the observation error in terms of norm $\|\cdot\|$ is less than $\delta$, we can estimate the prediction error by considering the following nonlinear optimization problem

$E_{u}=\max _{\left\|\boldsymbol{u}_{0}\right\| \leq \delta}\left\|M\left(\boldsymbol{U}_{0}+\boldsymbol{u}_{0}\right)(T)-M\left(\boldsymbol{U}_{0}\right)(T)\right\|$.

Mu et al. (2002) proved that $E_{u}$ is the upper bound of the prediction error, that is, $E \leq E_{u}$. The conditional nonlinear optimal perturbation $\boldsymbol{u}_{0 \delta}$ obtained from the Sect. 2 satisfies

$E_{u}=\left\|M\left(\boldsymbol{U}_{0}+\boldsymbol{u}_{0 \delta}\right)(T)-M\left(\boldsymbol{U}_{0}\right)(T)\right\|$.

Therefore, the CNOP and $J\left(\boldsymbol{u}_{0 \delta}\right)$ give an upper bound of the prediction error caused by the initial observational errors. 


\subsubsection{Estimation of prediction error of an El Niño event $\boldsymbol{U}^{(1)}(t)$}

The basic state, $\boldsymbol{U}^{(1)}(t)$, is an El Niño event (Fig. 4) in this model. For $T=10$ months, we obtain the CNOP of $\boldsymbol{U}^{(1)}(t)$ and the corresponding nonlinear evolution, which, as we have mentioned, gives an upper bound for the prediction error. It is found that, for constraint condition $\left\|\boldsymbol{u}_{0}\right\| \leq \delta$, $\delta \in[0.01,0.14]$, there exists a CNOP of $\boldsymbol{U}^{(1)}(t)$, which is on the boundary of disc $\left\|\boldsymbol{u}_{0}\right\| \leq \delta, \delta \in[0.01,0.14]$. Since $\boldsymbol{U}^{(1)}(0)$ is not the origin of the coordinate system, to define the position angle of CNOP on the plane $T_{E}-h_{E}$, we adopt a polar coordinate system, whose pole is $\boldsymbol{U}^{(1)}(0)$, and polar axis is parallel to the positive $T_{E}$-axis. Denote the position angle of the CNOP by $\theta_{N \delta}^{(1)}$, this CNOP can be expressed as $\left(\delta \cos \theta_{N \delta}^{(1)}, \delta \sin \theta_{N \delta}^{(1)}\right)$, where the position angle is the one between CNOP and the polar axis. Similar to the case of basic state $O$, for the basic state $\boldsymbol{U}^{(1)}(t)$, there are also notable differences between the nonlinear and the linear evolution of CNOP, and between the position angles of CNOPs and those of LSVs. The details are shown in Fig. 5.

For $T=12$ months, there also exists a CNOP $\left(\delta \cos \theta_{N \delta}^{(1)}, \delta \sin \theta_{N \delta}^{(1)}\right)$. The difference between the CNOP and the LSV are similar to the case of $T=10$ months. For simplicity, we do not show the results here.

\subsubsection{Estimation of prediction error of a La Niña event $\boldsymbol{U}^{(2)}$}

Similar to the case of the El Niño $\boldsymbol{U}^{(1)}(t)$, we compute the CNOPs of a La Niña event $\boldsymbol{U}^{(2)}(t)$ (Fig. 4), and compare them to the linear singular vectors of the corresponding tangent linear model. It is found that, for $\delta \in$ [0.01, 0.14] and $T=10,12$ months, there exists a CNOP $\left(\delta \cos \theta_{N \delta}^{(2)}, \delta \sin \theta_{N \delta}^{(2)}\right)$, respectively. Figure 6 shows the differences between the nonlinear and linear evolution, and between the position angles of CNOPs and those of LSVs for $T=12$ months. It follows that there is no significant difference when the initial perturbations are sufficient small. But with $\delta$ increasing from 0.01 to 0.14 , the difference becomes more and more distinguishable. Similarly, the position angles of LSV and CNOP of $\boldsymbol{U}^{(2)}(t)$ have considerable differences. All these suggest that in these cases the TLM is not a good approximation to the original nonlinear model. If TLM is used to estimate the error growth, the prediction error could be overestimated or underestimated, which yields uncertainty in the assessment of forecast skill. The usage of CNOP in the research of predictability is expected to provide improved results.

\section{Conclusion and discussion}

In this paper, we used a simple theoretical coupled oceanatmosphere model to demonstrate the concept of conditional nonlinear optimal perturbation (CNOP) and its application to study predictability problems of El Niño and La Niña events. It is shown that CNOP is more applicable than LSV in de-
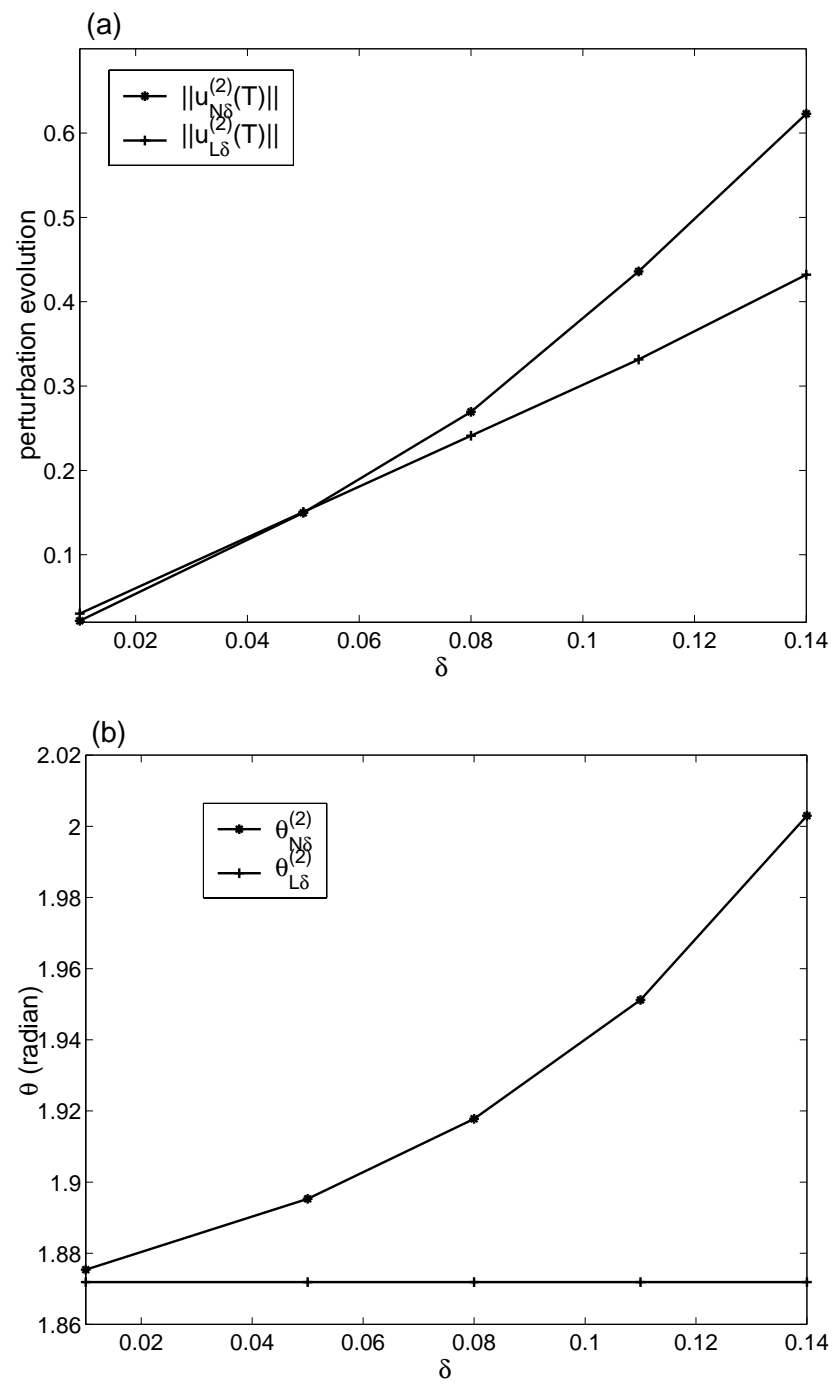

Fig. 6. (a) $\left\|\boldsymbol{u}_{N \delta}^{(2)}(T)\right\|$ and $\left\|\boldsymbol{u}_{L \delta}^{(2)}(T)\right\|$, the nonlinear and linear evolution of CNOP of $\boldsymbol{U}^{(2)}(t)$ for $T=12$ months and $\delta \in[0.01,0.14]$; (b) $\theta_{N \delta}^{(2)}$ and $\theta_{L \delta}^{(2)}$, the position angles of LSV and CNOP of $\boldsymbol{U}^{(2)}$ as functions of $\delta$, respectively.

termining the initial patterns that will evolve into El Niño or La Niña events most probably. In the case of estimation of prediction error, comparisons between CNOP and linear singular vector (LSV) suggest that CNOP be also a better tool than LSV.

There is an essential difference between LSV and CNOP. LSV represents the optimal growing direction of the initial perturbations in the TLM, CNOP stands for a kind of initial patterns, whose amplitude of the nonlinear evolution is maximal with the constraint condition at time $T$. Hence CNOP does represent the effects of model's nonlinearity. The physical explanation of CNOPs, and related observational data analysis are the works of the scientists in the future.

Although the model (Eq. 7) is a simple theoretical one, the characteristic of the nonlinearity of air-sea interaction is grasped, the nonlinear characteristic of the model is revealed 
from the aspects of the initial perturbation pattern and its corresponding nonlinear evolution by using the CNOP approach. It is reasonable to reckon that for more complicated nonlinear models governing the motions of the atmosphere and/or oceans, the results from CNOP approach could be significantly different from those from LSV approach. This indicates that it is worthwhile to survey the reliability of results obtained from the linear approach. The results of this study suggest that CNOP approach be one of useful tools in the study of nonlinear motions of atmosphere and oceans.

In this paper, we only calculate CNOP of two dimensional ordinary differential equations. Concerning the calculations of CNOP with high dimensional system, computational cost is of importance. For two-dimensional quasigeostrophic model with freedom of $10^{3}$, we have succeeded in obtaining CNOP by SQP method. The success of gaining CNOP numerically depends mostly on the optimization algorithm. If the algorithm is capable of obtaining a minimum, it is hopeful to get CNOP by this algorithm. Sometimes, parallel algorithm is a useful tool to capture the global minimum, considering that there exist multi-CPU computers. In operational 4-dimensional variational data assimilation (4-D-VAR) and ensemble forecast, computational cost, particularly the computer time, is of importance. But if we only consider the applications of CNOP to the research of predictability, the computer time is the secondary consideration.

For more complicated models employed in the numerical weather and climate prediction, the involved optimization problems could be difficult. The models are often of high dimensions, and the constraint conditions on physical variables or the observation errors can be complex. In some cases, the problems are non-smooth one too. To obtain CNOP of these models, we have to solve the optimization problems with complicated constraint conditions and with high dimensions. To overcome these difficulties, the collaborations between computational mathematicians and atmospheric and oceanic scientists are necessary. Nevertheless these difficulties are not the reason for stopping our investigation. Considering that operational 4-D-VAR has been successful implemental at the European Center for Medium-Range Weather Forecasts (ECMWF), which solve an optimization problem of dimension $10^{6}-10^{7}$, we are encouraged to expect that the rapid development of computational mathematics and computer will enable us to achieve our purpose step by step.

Acknowledgements. The authors are grateful to the anonymous reviewers for useful suggestion. This work was supported by KZCX2-208 of the Chinese Academy of Sciences, and the National Nature Scientific Foundation of China (Nos. 40233029, 40221503 and 40075015).

\section{References}

Aurell, E., Boffetta, G., Crisanti, A., Paladin, G., and Vulpiani, A.: Predictability in the large: An extension of the concept of Lypunov exponent, J. Phys. A30, 1-26, 1997.
Barclay, A., Gill, P. E., and Rosen, J. B.: SQP methods and their application to numerical optimal control, Numerical Analysis Report 97-3, Department of Mathematics, University of California, San Diego, La Jolla, CA, 1997.

Barkmeijer, J.: Constructing fast-growing perturbations for the nonlinear regime, J. Atmos. Sci., 53, 2838-2851, 1996.

Boffetta, G., Giuliani, P., Paladin, G., and Vulpiani, A.: An extension of the Lyapunov analysis for the predictability problem, J. Atmos. Sci., 55, 3409-3416, 1998.

Buizza, R. and Palmer, T. N.: The singular-vector structure of the atmospheric general circulation, J. Atmos. Sci., 52, 1434-1456, 1995.

Durbiano, S.: Vecteurs caracteristiques de modeles oceaniques pour la reduction d'ordre er assimilation de donnees, Ph.D. These, Université Joseph Fourier, Grenoble, 2001.

Lacarra, J.F. and Talagrand, O.: Short-range evolution of small perturbation in a baratropic model, Tellus, 40A, 81-95, 1988.

Lindzen, R. S. and Nigam, S.: On the role of sea surface temperature gradients in forcing low-level winds and convergence in the tropics, J. Atmos. Sci., 44, 2418-2436, 1987.

Lorenz, E. N.: Deterministic nonperiodic flow, J. Atmos. Sci., 20 , 130-141, 1963.

Lorenz, E. N.: A study of the predictability of a 28-variable atmospheric model, Tellus, 17, 321-333, 1965.

Lorenz, E. N.: Predictability - A problem partly solved. Proc. seminar on predictability, Reading, United Kindom, European Center for Medium-Range Weather Forecasts, 1-18, 1996.

$\mathrm{Mu}, \mathrm{M}$.: Nonlinear singular vectors and nonlinear singular values, Science in China (D), 43, 375-385, 2000.

Mu, M., Guo, H., Wang, J. F., and Li, Y.: The impact of nonlinear stability and instability on the validity of the tangent linear model, Adv. Atmos. Sci., 17, 375-390, 2000.

$\mathrm{Mu}, \mathrm{M}$. and Wang, J. C.: Nonlinear fastest growing perturbation and the first kind of predictability, Science in China (D), 44, 11281139, 2001.

Mu, M., Duan, W. S., and Wang, J. C.: The predictability problems in numerical weather and climate prediction, Adv. Atmos. Sci., 19, 191-204, 2002.

Oortwijn, J. and Barkmeijer, J.: Perturbations that optimally trigger weather regimes, J. Atmos. Sci., 52, 3932-3944, 1995.

Powell, M. J. D.: VMCWD: A FORTRAN subroutine for constrained optimization, DAMTP Report 1982/NA4, University of Cambridge, England, 1982.

Samelson, R. M. and Tziperman, E.: Instability of the chaotic ENSO: the growth-phase predictability barrier, J. Atmos. Sci., 58, 3613-3625, 2001.

Tanguay, M., Bartello, P., and Gauthier, P.: Four-dimensional data assimilation with a wide range of scales, Tellus, 47A, 974-997, 1995.

Thompson, C. J.: Initial conditions for optimal growth in a coupled ocean-atmosphere model of ENSO, J. Atmos. Sci., 55, 537-557, 1998.

Toth, Z. and Kalnay, E.: Ensemble forecasting at NCEP: the breeding method, Mon. Wea. Rev., 125, 3297-3318, 1997.

Wang, B. and Fang, Z.: Chaotic oscillation of the tropical climate: A dynamic system theory for ENSO, J. Atmos. Sci., 53, 27862802, 1996.

Wang, B., Barcilon, A., and Fang, Z.: Stochastic dynamics of El Niño-Southern Oscillation, J. Atoms. Sci., 56, 5-23, 1999.

Xue, Y., Cane, Y. M. A., and Zebiak, S. E.: Predictability of a coupled model of ENSO using singular vector analysis. Part I: Optimal growth in seasonal background and ENSO cycles, Mon. 
Wea. Rev., 125, 2043-2056, 1997a.

Xue, Y., Cane, Y. M. A., and Zebiak, S. E.: Predictability of a coupled model of ENSO using singular vector analysis. Part II: optimal growth and forecast skill, Mon. Wea. Rev., 125, 2057-2073, 1997b.
Zebiak, S. E. and Cane, M. A.: A model El Niño-Southern Oscillation, Mon. Wea. Rev., 115, 2262-2278, 1987. 